\title{
The Structure of Isomorphic Digraph from Powers Modulo $p^{e}$
}

\author{
Jinxing Zhao $\mathbb{D}^{1},{ }^{1}$ Guixin Deng $\mathbb{D},{ }^{2}$ and Tiejun Liu $\mathbb{D}^{3}$ \\ ${ }^{1}$ School of Mathematical Sciences, Inner Mongolia University, Hohhot 010021, China \\ ${ }^{2}$ College of Mathematics and Statistics, Guangxi Teachers Education University, Nanning 530023, China \\ ${ }^{3}$ Institute of Water Resources for Pastoral Ministry of Water Resources, Hohhot 010020, China \\ Correspondence should be addressed to Tiejun Liu; mksltj@126.com
}

Received 30 August 2018; Accepted 12 November 2018; Published 2 December 2018

Academic Editor: Andrei V. Kelarev

Copyright (C) 2018 Jinxing Zhao et al. This is an open access article distributed under the Creative Commons Attribution License, which permits unrestricted use, distribution, and reproduction in any medium, provided the original work is properly cited.

For positive integers $n$ and $k$, let $G(n, k)$ denote the digraph whose set of vertices is $\{0,1,2, \ldots, n-1\}$ and there is a directed edge from $a$ to $b$ if $a^{k} \equiv b(\bmod n)$. It is great value to consider conditions of $G\left(p, k_{1}\right) \simeq G\left(p, k_{2}\right)$. In this paper, we obtain necessary and sufficient conditions for $G\left(n, k_{1}\right) \simeq G\left(n, k_{2}\right)$ when $n=p^{e}$.

\section{Introduction}

Let $X$ be a nonempty set and $f$ be a map from $X$ to itself. Let $G(X, f)$ denote the digraph whose set of vertices is $X$ and there is a directed edge from $a \in X$ to $b \in X$ if $f(a)=b$. In the case that $X$ is a commutative ring or a finite abelian group (written multiplicative) and $f$ maps $x$ into its $k$-th power in $X$, we denote $G(X, f)$ by $G(X, k)$. In particular, let $G(n, k)$ denote the digraph whose set of vertices is $\mathbb{Z}_{n}=\{0,1,2, \ldots, n-1\}$ and there is a directed edge from $a$ to $b$ if $a^{k}=b$, or equivalently $a^{k} \equiv b(\bmod n)$. The investigation of graphs of this sort is important, in particular, because they have valuable applications, as explained, for example, in the book [1] and survey [2].

There are two particular subdigraphs of $G(n, k)$. Let $G_{1}(n, k)$ be the induced subdigraph of $G(n, k)$ on the set of vertices which are coprime to $n$ and $G_{2}(n, k)$ be the induced subdigraph of $G(n, k)$ on the set of vertices which are not coprime to $n$. We observe that $G_{1}(n, k)$ and $G_{2}(n, k)$ are disjoint and that $G(n, k)=G_{1}(n, k) \cup G_{2}(n, k)$. It is clear that $G_{1}(n, k) \simeq G\left(\mathbb{Z}_{n}^{*}, k\right)$, where $\mathbb{Z}_{n}^{*}$ is the unit group of $\mathbb{Z}_{n}$.

Many interesting properties of $G(n, k)$ are obtained by people. The distribution of indegree and structure of cycles of $G(n, k)$ are obtained in [3-9]. Somer and Krrížek [6, 10] determined when $G(n, k)$ is semiregular. Carlip and Mincheva [11] called a digraph $G$ symmetric of order $M$ if the component of $G$ can be partitioned into subsets of size $M$, each containing
$M$ isomorphic components. They also proved that $G(n, 2)$ is symmetric of order 2 for $n \equiv 2(\bmod 4)$ and $n \equiv 4(\bmod 8)$. Somer and Křížek obtained all $G(n, k)$ which are symmetric of order 2. Deng and Yuan [12] determined all $G(n, k)$ which are symmetric of order $M$, where $M$ has an odd prime divisor. Meemark and Wiroonsri $[13,14]$ investigated the structure of $G(R, k)$, where $R$ is the quotient ring of polynomials over finite fields.

In this paper, we are mainly interested in determining when $G\left(n, k_{1}\right) \simeq G\left(n, k_{2}\right)$. Lucheta, Miller, and Reiter [4] showed that $G\left(p, k_{1}\right)=G\left(p, k_{2}\right)$ if and only if $k_{1} \equiv$ $k_{2}(\bmod p-1)$. They also found that $G(11,2) \simeq G(11,8)$ and thus two digraphs $G\left(p, k_{1}\right)$ and $G\left(p, k_{2}\right)$ can be isomorphic without the condition. Wilson [9] also obtained that $G_{1}\left(n, k_{1}\right)=G_{1}\left(n, k_{2}\right)$ if and only if $k_{1} \equiv k_{2}(\bmod \lambda(n))$, where $\lambda$ is the Carmichael lambda-function. Deng and Yuan [15] gave a necessary and sufficient condition for $G\left(p, k_{1}\right) \simeq$ $G\left(p, k_{2}\right)$. They also determined that $G\left(H, k_{1}\right) \simeq G\left(H, k_{2}\right)$ for an abelian group $H$ by using graph theory and group theory. In this paper, we obtain a necessary and sufficient condition for $G\left(n, k_{1}\right) \simeq G\left(n, k_{2}\right)$.

This paper is organized as follows. In Section 2, we review some basic properties of $G(n, k)$ including indegrees, cycles, and structure of components. We point out that $G\left(\mathbb{Z}_{n}^{*}, k\right)$ is an induced subdigraph of $G(n, k)$. In Section 3, we review the properties of product of digraphs. In Section 4, we investigate $G(n, k)$ when $n$ is a power of a prime $p$ and obtain a necessary and sufficient condition for that $G\left(p^{e}, k_{1}\right) \simeq G\left(p^{e}, k_{2}\right)$. 


\section{Some Preliminary Results on $G(H, k)$ and $G(n, k)$}

In this section we review some basic properties of $G(n, k)$ and $G(H, k)$, where $H$ is a finite abelian group. The structure of $G(H, k)$ and hence $G_{1}(n, k)$ is well understood in [16]. It has highly symmetric structure.

Before proceeding further, we need to review some properties of the Carmichael lambda-function $\lambda(n)$ and the exponent of an abelian group.

Definition 1. Let $n$ be a positive integer. Then the Carmichaellambda-function $\lambda(n)$ is defined as follows:

$$
\begin{aligned}
\lambda(1) & =1, \\
\lambda(2) & =1, \\
\lambda(4) & =2, \\
\lambda\left(2^{k}\right) & =2^{k-2} \quad \text { for } k \geq 3 ; \\
\lambda\left(p^{k}\right) & =(p-1) p^{k-1}
\end{aligned}
$$

for any odd prime $p$ and $k \geq 1$;

$\lambda\left(p_{1}^{k_{1}} \ldots p_{r}^{k_{r}}\right)=\operatorname{lcm}\left[\lambda\left(p_{1}^{k_{1}}\right), \ldots, \lambda\left(p_{r}^{k_{r}}\right)\right]$, where $p_{1}, \ldots, p_{r}$ are distinct primes and $k_{i} \geq 1$ for all $i \in\{1,2, \ldots, r\}$.

The following theorem generalizes the well-known Euler's theorem which says that $a^{\phi(n)} \equiv 1(\bmod n)$ if and only if $\operatorname{gcd}(a, n)=1$.

Theorem 2 (Carmichael). Let $a, n \in \mathbb{N}$. Then $a^{\lambda(n)} \equiv$ $1(\bmod n)$ if and only if $\operatorname{gcd}(a, n)=1$. Moreover, there exists an integer $g$ such that ord $\mathrm{d}_{n} g=\lambda(n)$, where or $\mathrm{d}_{n} g$ denotes the multiplicative order of $g$ modulo $n$.

For the proof see [17, p. 21].

Let $H$ be a finite group. The exponent of $G$, denoted by $e(G)$, is the minimal positive integer $m$ such that $g^{m}=1$ for any $g \in H$. It is well known that if $n=\prod_{i=1}^{r} p_{i}^{e_{i}}$ is the prime factorization of $n$, then $\mathbb{Z}_{n} \simeq \mathbb{Z}_{p_{1}^{e_{1}}} \times \mathbb{Z}_{p_{2}^{e_{2}}} \times \cdots \times \mathbb{Z}_{p_{r}^{e_{r}}}$ and hence $\mathbb{Z}_{n}^{*} \simeq \mathbb{Z}_{p_{1}^{e_{1}}}^{*} \times \mathbb{Z}_{p_{2}^{e_{2}}}^{*} \times \cdots \times \mathbb{Z}_{p_{r}^{e_{r}}}^{*}$. Thus, $\lambda(n)$ is exactly the exponent of the unit group of $\mathbb{Z}_{n}$.

We have the following result about $G\left(H, k_{1}\right)$ and $G\left(H, k_{2}\right)$.

Theorem 3 ([16] Theorem 3.2-3.4). Let $H$ be an abelian group and let $e(H)$ be the exponent of $H$. Then $G\left(H, k_{1}\right) \simeq G\left(H, k_{2}\right)$ if and only if the following conditions hold:

(i) $\operatorname{gcd}\left(e(H), k_{1}\right)=\operatorname{gcd}\left(e(H), k_{2}\right)$,

(ii) we have a factorization of $e(H)=u v$, where $u$ is the maximal divisor of $e(H)$ relatively prime to $k_{1}$ and is also the maximal divisor of $e(H)$ relatively prime to $k_{2}$; for any divisor $d$ of $u$, ord $_{d} k_{1}=\operatorname{ord}_{d} k_{2}$.

Moreover, let $E_{i}$ be the component of $G\left(H, k_{i}\right)$ containing the identity element 1 of $H, i=1,2$. Condition ( $i$ ) is equivalent to $E_{1} \simeq E_{2}$.
Definition 4. We define a height function on the vertices and components of $G(n, k)$. Let $c$ be a vertex of $G(n, k)$. Then $h(c)$ is defined to be the minimal nonnegative integer $i$ such that $c^{k^{i}}$ is congruent modulo $n$ to a cycle vertex in $G(n, k)$. If $C$ is a component of $G(n, k)$, then we define $h(C)=\sup _{c \in C} h(c)$.

The indegree and height function play an important role in the structure of $G(n, k)$. We need the following results concerning the indegrees and heights.

Lemma 5 ([10] Theorem 3.1). Let $n=\prod_{i=1}^{r} p_{i}^{e_{i}}$ be the prime factorization of $n$. Let $a$ be a vertex of positive indegree in $G_{1}(n, k)$. Then

$$
\operatorname{indeg}_{n}^{k}(a)=\prod_{i=1}^{r} \operatorname{indeg}_{p_{i}^{e_{i}}}^{k}(a)=\prod_{i=1}^{r} \delta_{i} \operatorname{gcd}\left(\lambda\left(p_{i}^{e_{i}}\right), k\right),
$$

where $\delta_{i}=2$ if $2 \mid k$ and $8 \mid p_{i}^{e_{i}}$, and $\delta_{i}=1$ otherwise.

Lemma 6 ([10] Theorem 3.2). Let $p$ be a prime. Let a be a vertex with positive indegree in $G_{2}\left(p^{e}, k\right)$. Suppose that $a \neq 0$ and $p^{l} \| a$. Then $l=k t$ for some positive integer $t$ and

$$
\operatorname{indeg}_{p^{e}}^{k}(a)=\delta p^{(k-1) t} \operatorname{gcd}\left(\lambda\left(p^{e-l}\right), k\right),
$$

where $\delta=2$ if $p=2,2 \mid k$ and $e-l \geq 3$, and $\delta=1$ otherwise.

Lemma 7 ([18] Lemma 3.2). Let $p$ be a prime and e, $k$ be two positive integers. Then

$$
\operatorname{indeg}_{p^{e}}^{k}(0)=p^{e-\lceil e / k\rceil}
$$

Lemma 8. Let $p$ be a prime and $e \geq 2, k \geq 2$ be two positive integers. Let $h$ be the unique positive integer such that $k^{h-1}<$ $e \leq k^{h}$. Then $h=h\left(G_{2}\left(p^{e}, k\right)\right)$.

Proof. It is clear that $p \in G_{2}\left(p^{e}, k\right)$ and $h(p)=h\left(G_{2}\left(p^{e}, k\right)\right)$. Moreover, $p^{k^{i}} \equiv 0\left(\bmod p^{e}\right)$ if and only if $k^{i} \geq e$. The proof is complete.

Lemma 9. Let $p$ be a prime and $e, k \geq 2$ be two positive integers. Let $\lambda\left(p^{e}\right)=u v$, where $u$ is the maximal divisor of $\lambda\left(p^{e}\right)$ relatively prime to $k$. If $C$ is the component of $G\left(p^{e}, k\right)$ containing 1 , then

$$
h(C)=\min \left\{i: v \mid k^{i}\right\}
$$

Proof. Let $h=\min \left\{i: v \mid k^{i}\right\}$. Then there exists a divisor $d$ of $v$ such that $d$ is not a divisor of $k^{h-1}$. By Theorem 2, there exists a vertex $g \in G\left(p^{e}, k\right)$ such that ord $p_{p^{e}} g=u v$. Let $a \equiv$ $g^{u v / d}\left(\bmod p^{e}\right)$. Then $\operatorname{ord}_{p^{e}} a=d, a^{k^{h-1}} \not \equiv 1\left(\bmod p^{e}\right)$, and $a^{k^{h}} \equiv 1\left(\bmod p^{e}\right)$. Hence, $h(C) \geq h(a)=h$ by the definition of the height function.

Conversely, if $a \in C$, then there exists $j \geq 1$ such that $a^{k^{j}} \equiv 1\left(\bmod p^{e}\right)$, so ord $p_{p^{e}} a \mid k^{j}$. Since $\operatorname{ord}_{p^{e}} a \mid u v$, we have $\operatorname{ord}_{p^{e}} a \mid v$, so $a^{k^{h}} \equiv 1\left(\bmod p^{e}\right)$. That is, $h(C) \leq h$. 


\section{Properties of Digraphs Products}

Given two digraphs $G_{1}$ and $G_{2}$. Let $G_{1} \times G_{2}$ denote the digraph whose vertices are the ordered pairs $\left(a_{1}, a_{2}\right)$, where $a_{i} \in G_{i}$ and there is a directed edge from $\left(a_{1}, a_{2}\right)$ to $\left(b_{1}, b_{2}\right)$ if there is a directed edge from $a_{1}$ to $b_{1}$ and a directed edge from $a_{2}$ to $b_{2}$. Somer and Kř́žek [18] noted the following fact: if $n=n_{1} n_{2}$ and $\operatorname{gcd}\left(n_{1}, n_{2}\right)=1$, then $G(n, k) \cong G\left(n_{1}, k\right) \times G\left(n_{2}, k\right)$. The canonical isomorphism is given by $a \longmapsto\left(a_{1}, a_{2}\right)$ where $a \equiv$ $a_{i}\left(\bmod n_{i}\right), i=1,2$. In general,

$$
G(n, k) \cong G\left(p_{1}^{e_{1}}, k\right) \times G\left(p_{2}^{e_{2}}, k\right) \times \cdots \times G\left(p_{r}^{e_{r}}, k\right),
$$

if $n=\prod_{i=1}^{r} p_{i}^{e_{i}}$ is the prime factorization of $n$. We use the following notation:

$$
\prod_{i=1}^{r} G_{i}:=G_{1} \times G_{2} \times \cdots \times G_{r}
$$

where each $G_{i}$ is a digraph. We need this fact and the following lemmas.

Lemma 10 ([19] Lemma 3.1). Let $n=n_{1} n_{2}$ where $\operatorname{gcd}\left(n_{1}, n_{2}\right)=1$. Let $E_{i}$ be a component of $G\left(n_{i}, k\right)$ with cycle length $t_{i}$. Then $E_{1} \times E_{2}$ is a subdigraph of $G(n, k)$ consisting of $\operatorname{gcd}\left(t_{1}, t_{2}\right)$ components, each having cycles of length $1 \mathrm{~cm}\left[t_{1}, t_{2}\right]$.

Corollary 11. Let $G_{1}, G_{2}$, and $G_{3}$ be digraphs. Suppose that $A_{t}\left(G_{1} \times G_{3}\right)=A_{t}\left(G_{2} \times G_{3}\right)$. Then $A_{t}\left(G_{1}\right)=A_{t}\left(G_{2}\right)$.

Proof. Let $a_{i}$ be the number of $i$-cycles contained in $G_{3}, b_{i}$ be the number of $i$-cycles contained in $G_{1}$, and $c_{i}$ be the number of $i$-cycles contained in $G_{2}$. Using Lemma 10 to compare the number of $l$-cycles of $G_{i} \times G_{3}$, we have

$$
\sum_{1 \mathrm{~cm}[i, j]=l} a_{i} b_{j} \operatorname{gcd}(i, j)=\sum_{1 \mathrm{~cm}[i, j]=l} a_{i} c_{j} \operatorname{gcd}(i, j) .
$$

Let $l=1$; we see that $b_{1}=c_{1}$. Now assume that $b_{i}=c_{i}$ for any $i<l$. We have

$$
\sum_{i \mid l} a_{i} b_{l} \operatorname{gcd}(i, l)=\sum_{i \mid l} a_{i} c_{l} \operatorname{gcd}(i, l) .
$$

Thus $b_{l}=c_{l}$ and we finish the proof by induction.

Lemma 12 ([19] Lemma 3.12). Let $n=\prod_{i=1}^{r} p_{i}^{e_{i}}$ be the prime factorization of $n$. Let $a=\left(a_{1}, a_{2}, \ldots, a_{r}\right)$ and $b=$ $\left(b_{1}, b_{2}, \ldots, b_{r}\right)$ be two vertices in $G(n, k) \cong G\left(p_{1}^{e_{1}}, k\right) \times$ $G\left(p_{2}^{e_{2}}, k\right) \times \cdots \times G\left(p_{r}^{e_{r}}, k\right)$. If $a$ and $b$ are in the same cycle, then $a_{i}$ and $b_{i}$ are in the same cycle for each $i$.

Let $|G|$ denote the number of vertices of a digraph G. Let $M(G)=\max _{c \in G}\{$ indeg $(c)\}, N(G)=$ $\min _{c \in G, \text { indeg }(c)>0}\{$ indeg $(c)\}$, and $I(G)=\mid\{$ indeg $(a): a \in$ $G\} \backslash\{0\} \mid$. The following lemma is immediately from the properties of product of digraphs.

Lemma 13. Let $G_{1}$ and $G_{2}$ be two digraphs, and $a_{1} \in G_{1}$ and $a_{2} \in G_{2}$. Then $\alpha=\left(a_{1}, a_{2}\right) \in G \times H$. Moreover, indeg $(\alpha)=$ indeg $\left(a_{1}\right)$ indeg $\left(a_{2}\right), M\left(G_{1} \times G_{2}\right)=M\left(G_{1}\right) M\left(G_{2}\right), N\left(G_{1} \times G_{2}\right)$ and $\left|G_{1} \times G_{2}\right|=\left|G_{1}\right|\left|G_{2}\right|$. If $I\left(G_{1}\right) \geq 2$ and $I\left(G_{2}\right) \geq 2$, then $I\left(G_{1} \times G_{2}\right)>\max \left\{I\left(G_{1}\right), I\left(G_{2}\right)\right\}$.
Definition 14. For any positive integers $t$ and $m$, we define $O_{t}^{m}$ to be the digraph which satisfies the following: (i) it has $t m$ vertices and a $t$-cycle, (ii) indeg $(a)=m$ if $a$ is a cycle vertex and indeg $(a)=0$ otherwise. In particular, let $O_{t}=O_{t}^{1}$ be the $t$-cycle.

Remark 15. Let $C$ be a component of $G(n, k)$ with $h(C) \leq 1$. By Lemma 12, each cycle vertex of $C$ has the same indegree. Thus, $C \simeq O_{t}^{m}$, where $t$ is the cycle length of $C$ and $m$ is the indegree of a cycle vertex of $C$. We use cyindeg $(C)$ to denote the indegree of a cycle vertex of $C$.

\section{The Main Result When $n=p^{e}$}

In this section we study $G\left(p^{e}, k\right)$, where $p$ is prime. It is clear that $G_{2}\left(p^{e}, k\right)$ consists of a single component. We begin with the following Lemma.

Lemma 16. Let $p$ be an odd prime and $e, k$ two positive integers. Suppose that $\operatorname{gcd}\left((p-1) p^{e-1}, k\right) \geq p^{e-1}$. Then

$$
G\left(p^{e}, k\right) \simeq O_{1}^{p^{e-1}} \times G(p, k) .
$$

Proof. Let $C_{i}$ denote the cyclic group of order $i$. Recall that for an abelian group $H, G(H, k)$ is the digraph whose vertex set is $H$. There exists a directed edge from $a$ to $b$ if and only if $b=a^{k}$. Note that the unit group $\mathbb{Z}_{p^{e}}$ is a cyclic group of order $(p-1) p^{e-1}$. Thus, $G_{1}\left(p^{e}, k\right) \simeq G\left(C_{(p-1) p^{e-1}}, k\right) \simeq G\left(C_{p-1}, k\right) \times$ $G\left(C_{p^{e-1}}, k\right)$ for any $e \geq 1$.

By hypothesis we have $p^{e-1} \mid k$, and hence $G\left(C_{p^{e-1}}, k\right) \simeq$ $O_{1}^{p^{e-1}}$. Since $G(p, k) \simeq O_{1} \cup G\left(C_{p-1}, k\right)$, we have

$$
\begin{aligned}
G\left(p^{e}, k\right) & \simeq G_{1}\left(p^{e}, k\right) \cup G_{2}\left(p^{e}, k\right) \\
& \simeq\left(G\left(C_{p-1}, k\right) \times G\left(C_{p^{e-1}}, k\right)\right) \cup O_{1}^{p^{e-1}} \\
& \simeq\left(O_{1}^{p^{e-1}} \times G\left(C_{p^{e-1}}, k\right)\right) \cup\left(O_{1}^{p^{e-1}} \times O_{1}\right) \\
& \simeq O_{1}^{p^{e-1}} \times\left(O_{1} \cup G\left(C_{p-1}, k\right)\right) \\
& \simeq O_{1}^{p^{e-1}} \times G(p, k) .
\end{aligned}
$$

Recall that a digraph $G$ is called semiregular if there exists a positive integer $d$ such that each vertex of $G$ has either indegree $d$ or 0 . In this case, $G$ is called $d$-semiregular.

Lemma 17 ([10] Theorem 4.4). Let $p$ be a prime and e, $k \geq 2$ two positive integers.

(1) If $p$ is odd, then we can obtain that $G_{2}\left(p^{e}, k\right)$ is semiregular if and only if $e \leq k$ or $\operatorname{gcd}(p-1, k)=1$ and $e \leq k+r+1$, where $p^{r} \| k$. In the first case $G_{2}\left(p^{e}, k\right)$ is $p^{e-1}$-semiregular, and in the second case $G_{2}\left(p^{e}, k\right)$ is $p^{e-2}$-semiregular. Thus, $G\left(p^{e}, k\right)$ is semiregular if and only if $\operatorname{gcd}\left(\lambda\left(p^{e}\right), k\right)=p^{e-1}$.

(2) If $p=2$, then $G_{2}\left(2^{e}, k\right)$ is semiregular if and only if one of the following conditions hold: 
(a) $k=2$ and $e \in\{1,2,3,4,6\}$;

(b) $k=4$ and $1 \leq e \leq 9$;

(c) $k \geq 6$ and $1 \leq e \leq k+r+2$, where $2^{r} \| k$.

Moreover, $G\left(2^{e}, k\right)$ is semiregular if and only if one of the following conditions hold:
(a) $k=2$ and $e \in\{1,2,4\}$;
(b) $k=4$ and $1 \leq e \leq 5$;
(c) $k \geq 6$ and $1 \leq e \leq r+2$, where $2^{r} \| k$.

Lemma 18. Let $p$ be a prime. Suppose that $\operatorname{gcd}(p-1, k)=$ 1. Then for any positive integers $e \geq 1, k \geq 2$, we have indeg $_{p^{e}}^{k}(0) \geq$ indeg $_{p^{e}}^{k}(1)$, except the case $(p, e, k)=(2,3,2)$.

Proof. In fact, for $e \leq k$, we have indeg $p^{e}(0)=p^{e-1}$ and indeg $g_{p^{e}}^{k}(1)=\delta \operatorname{gcd}\left(\lambda\left(p^{e}\right), k\right)$, where $\delta=2$ if $2 \mid k$ and $8 \mid p^{e}$, and $\delta=1$ otherwise. It is clear that indeg $g_{p^{e}}^{k}(0) \geq$ indeg $_{p^{e}}^{k}(1)$, since $\operatorname{gcd}(p-1, k)=1$.

Now, let $e>k$. Then $e \geq 3$. Assume that $p$ is odd. Suppose that $p^{r} \| k$. Let $s=\lceil e / k\rceil$. Then $(s-1) k<e \leq s k$. If $s \geq 3$, then $e-s \geq(s-1) k-s+1=(s-1)(k-1) \geq 2(k-1) \geq k \geq r$. Then indeg(0) $=p^{e-s} \geq p^{r}=$ indeg(1). If $s=2$, we still have $e-s=e-2 \geq r$. Otherwise we have $p^{e-1} \mid k$. Since $e \geq 3$, then $e>k \geq p^{e-1}$, which is a contradiction.

Suppose that $p=2$. By Lemmas 5 and 7 , we know that $2=$ indeg $g_{8}^{2}(0)<$ indeg $g_{8}^{2}(1)=4$. In the following, we assume that $e \geq 4$. Suppose that $2^{r} \| k$. Let $s=\lceil e / k\rceil$. Then $(s-1) k<$ $e \leq s k$. If $s \geq 3$, then $e-s \geq(s-1) k-s+1=(s-1)(k-1) \geq$ $2(k-1) \geq k \geq r+1$. Then indeg $(0)=2^{e-s} \geq 2^{r+1} \geq$ indeg $(1)$. If $s=2$, by easy computations we still have $e-s=e-2 \geq r+1$ since $e>k$.

We use the following notation. Let $p$ be a fixed prime. Let the mapping $v_{p}$ be defined by $v_{p}: \mathbb{N}^{*} \longrightarrow \mathbb{N}$ such that $v_{p}(n)=\max \left\{m \geq 0: p^{m} \mid n\right\}$ for $n \in \mathbb{N}^{*}$, where $\mathbb{N}$ is the set of nonnegative integers and $\mathbb{N}^{*}$ is the set of positive integers. Furthermore, we define a function $V_{p}$ from the set of digraphs to the set of subsets of $\mathbb{N}$ as follows:

$$
V_{p}(G):=\left\{v_{p}(\operatorname{indeg}(c)): c \in G, \operatorname{indeg}(c)>0\right\}
$$

for any digraph $G$. Let $A$ and $B$ be two nonempty subsets of $\mathbb{N}$. We denote $A+B=\{a+b: a \in A, b \in B\}$. Since $v_{p}(m n)=$ $v_{p}(m)+v_{p}(n)$, we have $V_{p}(G \times H)=V_{p}(G)+V_{p}(H)$ for any digraphs $G$ and $H$.

Lemma 19. Let $p$ be a prime and e, $2 \leq k_{2} \leq k_{1}$ be positive integers. Suppose that $V_{p}\left(G_{2}\left(p^{e}, k_{1}\right)\right)=V_{p}\left(G_{2}\left(p^{e}, k_{2}\right)\right)$ and $v_{p}\left(\operatorname{gcd}\left(\lambda\left(p^{e}\right), k_{1}\right)\right)=v_{p}\left(\operatorname{gcd}\left(\lambda\left(p^{e}\right), k_{2}\right)\right)=r$. Then $e \leq$ $\min \left\{k_{1}, k_{2}\right\}$ or $k_{1}=k_{2}$.

Proof. If $e \leq \min \left\{k_{1}, k_{2}\right\}$, then $V_{p}\left(G_{2}\left(n, k_{1}\right)\right)=V_{p}\left(G_{2}(n\right.$, $\left.\left.k_{2}\right)\right)=\{e-1\}$ since $a^{k_{1}} \equiv a^{k_{2}} \equiv 0\left(\bmod p^{e}\right)$ for any $a \mid p$. Suppose that $k_{2} \leq e<k_{1}$. Then $e-1 \in V_{p}\left(G_{2}\left(p^{e}, k_{2}\right)\right)$. But $e-1 \notin V_{p}\left(G_{2}\left(p^{e}, k_{1}\right)\right)$. It contradicts the fact that
$V_{p}\left(G_{2}\left(n, k_{1}\right)\right)=V_{p}\left(G_{2}\left(n, k_{2}\right)\right)$. In the following we always assume that $e>k_{1} \geq k_{2}$.

Case 1. First we assume that $p$ is an odd prime. We show that $\left\lceil e / k_{1}\right\rceil=\left\lceil e / k_{2}\right\rceil$. Let $\left\lceil e / k_{i}\right\rceil=s_{i}$. We need to show that $\max \left\{V_{p}\left(G_{2}\left(p^{e}, k_{i}\right)\right)\right\}=e-s_{i}$. By Lemmas 5, 6, and 7, we see that

$$
\begin{aligned}
& V_{p}\left(G_{2}\left(p^{e}, k_{i}\right)\right)=\left\{\left(k_{i}-1\right) t+\min \left\{e-k_{i} t-1, r\right\}: t\right. \\
& \left.\quad=1,2, \ldots, s_{i}-1\right\} \cup\left\{0, e-s_{i}\right\} .
\end{aligned}
$$

We have $\left(s_{i}-1\right) k_{i}<e \leq s_{i} k_{i}$, and then $e-k_{i} t-1 \geq k_{i}>r$ for any $t<s_{i}-1$. Hence

$$
\begin{aligned}
& \left(k_{i}-1\right)\left(s_{i}-1\right)-\left(k_{i}-1\right) t-\min \left\{e-k_{i} t-1, r\right\} \\
& \quad=\left(k_{i}-1\right)\left(s_{i}-t-1\right)-r \geq 0
\end{aligned}
$$

for any $1 \leq t<s_{i}-1$. It implies that

$$
\begin{aligned}
& \max \left\{V_{p}\left(G_{2}\left(p^{e}, k_{i}\right)\right)\right\}=\max \left\{\left(k_{i}-1\right)\left(s_{i}-1\right)\right. \\
& \left.+\min \left\{e-k_{i}\left(s_{i}-1\right)-1, r\right\}, e-s_{i}\right\} .
\end{aligned}
$$

But $\left(k_{i}-1\right)\left(s_{i}-1\right)+\min \left\{e-k_{i}\left(s_{i}-1\right)-1, r\right\} \leq\left(k_{i}-1\right)\left(s_{i}-\right.$ $1)+\left(e-k_{i}\left(s_{i}-1\right)-1\right)=e-s_{i}$. So $\max \left\{V_{p}\left(G_{2}\left(p^{e}, k_{i}\right)\right)\right\}=e-s_{i}$. We get $s_{1}=s_{2}$.

Now if $s_{1}=s_{2} \geq 3$, by (13) we have $\min \left\{V_{p}\left(G_{2}\left(p^{e}\right.\right.\right.$, $\left.\left.\left.k_{i}\right)\right) \backslash\{0\}\right\}=k_{i}-1+r$. Then $k_{1}-1+r=k_{2}-1+r, k_{1}=k_{2}$. If $s_{1}=$ $s_{2}=2$, then $\min \left\{V_{p}\left(G_{2}\left(p^{e}, k_{i}\right)\right) \backslash\{0\}\right\}=k_{i}-1+\min \left\{e-k_{i}-1, r\right\}$. We have $k_{1}-1+\min \left\{e-k_{1}-1, r\right\}=k_{2}-1+\min \left\{e-k_{2}-1, r\right\}$.

Subcase 1. If $r \leq e-k_{2}-1 \leq e-k_{1}-1$, then $k_{1}-1+r=k_{2}-1+r$ and $k_{1}=k_{2}$.

Subcase 2. If $e-k_{2}-1 \leq r \leq e-k_{1}-1$, then $k_{1}-1+r=$ $k_{2}-1+e-k_{2}-1=e-2$ and $e=k_{1}+r+1$. But we have $e>k_{2}$ and $p^{r} \mid k_{i}, i=1,2$. Let $k_{1}=p^{r} m_{1}, k_{2}=p^{r} m_{2}$. We have

$$
k_{2}-k_{1}=p^{r}\left(m_{2}-m_{1}\right)<r+1 \text {. }
$$

We get $m_{2}=m_{1}$ from this equation and thus $k_{1}=k_{2}$.

Subcase 3. If $e-k_{2}-1 \leq e-k_{1}-1 \leq r$, we have $k_{2}<e \leq k_{1}+r+1$. Using the same notation we still have (16); thus $k_{1}=k_{2}$.

Case 2. Now we treat the case $p=2$. The case $e \leq 3$ is trivial. Assume that $e \geq 4$; in this case we still have $\left\lceil e / k_{1}\right\rceil=\left\lceil e / k_{2}\right\rceil$. Let $\left\lceil e / k_{i}\right\rceil=s_{i}$. It suffices to show that $\max V_{2}\left(G_{2}\left(2^{e}, k_{i}\right)\right)=$ $e-s_{i}$. By Lemmas 5, 6, and 7, we see that

$$
\begin{aligned}
& V_{2}\left(G\left(2^{e}, k_{i}\right)\right)=\left\{v_{2}\left(\delta 2^{\left(k_{i}-1\right) t} \operatorname{gcd}\left(\lambda\left(2^{e-k_{i} t}\right), k_{i}\right)\right): t\right. \\
& \left.=1,2, \ldots, s_{i}-1\right\} \cup\left\{0, e-s_{i}\right\},
\end{aligned}
$$

where $\delta=2$ if $2 \mid k_{i}$ and $e-k_{i} t \geq 3$, and $\delta=1$ otherwise. If $r=0$, then $\max \left\{V_{2}\left(G_{2}\left(p^{e}, k_{i}\right)\right)\right\}=\max \left\{e-s_{i},\left(k_{i}-1\right)\left(s_{i}-1\right)\right\}=$ $e-s_{i}$. We have $s_{1}=s_{2}$. Suppose that $r>0$. Then $2 \mid k_{i}$. If 
$t<s_{i}-1, e-k_{i} t>\left(s_{i}-1\right) k_{i}-k_{i} t=\left(s_{i}-t-1\right) k_{i} \geq \max \{r, 3\}$. So

$$
\begin{aligned}
& V_{2}\left(G\left(2^{e}, k_{i}\right)\right)=\left\{1+\left(k_{i}-1\right) t+r: t=1,2, \ldots, s_{i}\right. \\
& \quad-2\} \cup\left\{0, e-s_{i}, \epsilon+\left(k_{i}-1\right)\left(s_{i}-1\right)\right. \\
& \left.\quad+v_{2}\left(\operatorname{gcd}\left(\lambda\left(2^{e-k_{i}\left(s_{i}-1\right)}\right), k_{i}\right)\right)\right\},
\end{aligned}
$$

where $\epsilon=1$ if $e-k_{i}\left(s_{i}-1\right) \geq 3$, and $\epsilon=0$ otherwise. We have

$$
\epsilon+v_{2}\left(\operatorname{gcd}\left(\lambda\left(2^{e-k_{i}\left(s_{i}-1\right)}\right), k_{i}\right)\right) \leq e-k_{i}\left(s_{i}-1\right)-1 .
$$

Thus $\max \left\{V_{2}\left(G_{2}\left(p^{e}, k_{i}\right)\right)\right\}=e-s_{i}, s_{1}=s_{2}$.

Now if $s_{1}=s_{2} \geq 3$, we have $\min \left\{V_{2}\left(G_{2}\left(2^{e}, k_{i}\right)\right) \backslash\{0\}\right\}=$ $1+k_{i}-1+r=k_{i}+r$. Then $k_{1}+r=k_{2}+r, k_{1}=k_{2}$. If $s_{1}=s_{2}=2$, then $\min \left\{V_{2}\left(G_{2}\left(2^{e}, k_{i}\right)\right) \backslash\{0\}\right\}=\epsilon_{i}+k_{i}-1+$ $v_{2}\left(\operatorname{gcd}\left(\lambda\left(2^{e-k_{i}}\right), 2^{r}\right)\right)$, where $\epsilon_{i}=1$ if $e-k_{i} \geq 3$, and $\epsilon=0$ otherwise. Assume that $k_{1} \leq k_{2}$.

Subcase $1.3 \leq e-k_{2} \leq e-k_{1}$. Then, we have $k_{1}+\min \left\{e-k_{1}-\right.$ $2, r\}=k_{2}+\min \left\{e-k_{2}-2, r\right\}$. $k_{1}=k_{2}$.

(i) If $r \leq e-k_{2}-2 \leq e-k_{1}-2$, then $k_{1}+r=k_{2}+r$,

(ii) If $e-k_{2}-2 \leq r \leq e-k_{1}-2$, then $e-2=k_{1}+r$. We have $k_{2}<e=k_{1}+r+2$, let $k_{i}=2^{r} m_{i}, i=1,2$. Then

$$
2^{r}\left(m_{2}-m_{1}\right)<r+2 .
$$

We get $r=0,1, m_{2}=m_{1}+1$. If $r=0$, then $k_{2}=k_{1}+1$. That is, one of $k_{1}, k_{2}$ is odd and the other one is even. It is contrary to $\operatorname{gcd}\left(\lambda\left(2^{e}\right), k_{1}\right)=\operatorname{gcd}\left(\lambda\left(2^{e}\right), k_{2}\right)=2^{r}$. If $r=1$, we have $4 \mid k_{1}$ or $4 \mid k_{2}$ and $\operatorname{gcd}\left(2^{e-2}, k_{1}\right)=\operatorname{gcd}\left(2^{e-2}, k_{2}\right)=2$. It implies that $e-2=1, e=3$. But it is contrary to $3 \leq e-k_{2}$.

(iii) If $e-k_{2}-2 \leq e-k_{1}-2 \leq r$, then $k_{2}<k_{1}+r+2$. Using the same notations we still have (20). It is impossible.

Subcase 2. $e-k_{2}<3 \leq e-k_{1}$. Then we have $k_{1}+\min \left\{e-k_{1}-\right.$ $2, r\}=k_{2}+\min \left\{e-k_{2}-1, r\right\}$. $k_{1}=k_{2}$.

(i) If $r \leq e-k_{2}-1$ and $r \leq e-k_{1}-2$, then $k_{1}+r=k_{2}+r$,

(ii) If $e-k_{2}-1 \leq r$ and $r \leq e-k_{1}-2$, then $e-1=k_{1}+r$. We have $k_{2}<e=k_{1}+r+1$. Let $k_{i}=2^{r} m_{i}, i=1,2$. Then

$$
2^{r}\left(m_{2}-m_{1}\right)<r+1 \text {. }
$$

We must have $m_{2}=m_{1}, k_{2}=k_{1}$.

(iii) If $e-k_{2}-1 \leq r$ and $e-k_{1}-2 \leq r$, then $e-1=e-2$. It is a contradiction.

Subcase 3. $e-k_{2} \leq e-k_{1}<3$. We have $k_{1}+\min \left\{e-k_{1}-1, r\right\}=$ $k_{2}+\min \left\{e-k_{2}-1, r\right\}$.

(i) If $e=k_{1}+2$, then $k_{1} \leq k_{2}<e=k_{1}+2, k_{2}=k_{1}+1$. One of $k_{1}$ and $k_{2}$ is odd and the other one is even. It is contrary to $\operatorname{gcd}\left(\lambda\left(2^{e}\right), k_{1}\right)=\operatorname{gcd}\left(\lambda\left(2^{e}\right), k_{2}\right)=2^{r}$.

(ii) If $e=k_{1}+1$, then $k_{1} \leq k_{2}<e=k_{1}+1$, and we still have $k_{2}=k_{1}$. The lemma is proved.

Corollary 20. Let $p$ be a prime and e be a positive integer. Let $k_{1} \geq 2, k_{2} \geq 2$ be two positive integers. Then $G\left(p^{e}, k_{1}\right) \simeq G\left(p^{e}, k_{2}\right)$ if and only if $G_{1}\left(p^{e}, k_{1}\right) \simeq G_{1}\left(p^{e}, k_{2}\right)$ and $G_{2}\left(p^{e}, k_{1}\right) \simeq G_{2}\left(p^{e}, k_{2}\right)$.
Proof. Suppose that $G\left(p^{e}, k_{1}\right) \simeq G\left(p^{e}, k_{2}\right)$. It suffices to show that $G_{2}\left(p^{e}, k_{1}\right) \simeq G_{2}\left(p^{e}, k_{2}\right)$. If $e \leq 2$, then $G_{2}\left(p^{e}, k_{1}\right) \simeq$ $G_{2}\left(p^{e}, k_{2}\right) \simeq O_{1}^{p}$. We assume that $e \geq 3$ and $p \geq 3$. If $G_{2}\left(p^{e}, k_{1}\right) \neq G_{2}\left(p^{e}, k_{2}\right)$, by Theorem 3 we have $G_{2}\left(p^{e}, k_{1}\right) \simeq$ $C_{2}$ and $G_{2}\left(p^{e}, k_{2}\right) \simeq C_{1}$ where $C_{i}$ is the component of $G\left(p^{e}, k_{i}\right)$ containing the vertex 1 . By Lemma 17 we have $\operatorname{gcd}\left((p-1) p^{e-1}, k_{1}\right)=p^{e-2}$ or $p^{e-1}, \operatorname{gcd}\left((p-1) p^{e-1}, k_{2}\right)=p^{e-2}$ or $p^{e-1}$. It implies that $p^{e-2} \mid k_{i}$ and therefore $e \leq p^{e-2} \leq k_{i}$ since $e \geq 3$. We have $G_{2}\left(p^{e}, k_{1}\right) \simeq G_{2}\left(p^{e}, k_{2}\right) \simeq O_{1}^{p^{e-1}}$, which is a contradiction.

If $p=2, e=3$, one of $k_{1}, k_{2}$ is 2 . Then by easy computations we know that $G\left(n, k_{1}\right) \simeq G\left(n, k_{2}\right)$ if and only if $k_{1}=k_{2}$. Now let $e \geq 4$; we have that $C_{1}, C_{2}, G_{2}\left(n, k_{1}\right)$ and $G_{2}\left(n, k_{2}\right)$ are semiregular. Then we have

$$
\begin{aligned}
\operatorname{indeg}_{2^{e}}^{k_{1}}(0) & =\operatorname{indeg}_{2^{e}}^{k_{2}}(1) \\
\text { and indeg } \ln _{2^{e}}^{k_{2}}(0) & =\operatorname{indeg}_{2^{e}}^{k_{1}}(1) .
\end{aligned}
$$

By Lemma 18,

$$
\begin{array}{r}
\operatorname{indeg}_{2^{e}}^{k_{1}}(0) \geq \operatorname{indeg}_{2^{e}}^{k_{1}}(1) \\
\text { and indeg } \text { 2 }_{2^{e}}^{k_{2}}(0) \geq \operatorname{indeg}_{2^{e}}^{k_{2}}(1) .
\end{array}
$$

Combining these formulas, we obtain $d=\operatorname{indeg}_{2^{e}}^{k_{1}}(0)=$ indeg $2_{2^{e}}^{k_{1}}(1)=\operatorname{indeg}_{2^{e}}^{k_{2}}(0)=\operatorname{indeg}_{2^{e}}^{k_{1}}(1)$ and $G\left(n, k_{i}\right)$ is $d$ semiregular, $i=1,2$.

If $e \leq \min \left\{k_{1}, k_{2}\right\}$, then $G_{2}\left(2^{e}, k_{1}\right) \simeq G_{2}\left(2^{e}, k_{2}\right) \simeq O_{1}^{d}$. If $e>\min \left\{k_{1}, k_{2}\right\}$, by Lemma 17 we know that $k_{1}=k_{2}$. We still have $G_{2}\left(2^{e}, k_{1}\right) \simeq G_{2}\left(2^{e}, k_{2}\right)$. The other direction is trivial.

Theorem 21. Let $p$ be an odd prime and e, $k_{1} \geq 2, k_{2} \geq 2$ positive integers. Then $G\left(p^{e}, k_{1}\right) \simeq G\left(p^{e}, k_{2}\right)$ if and only if one of the following conditions holds:

(i) If $e>\min \left\{k_{1}, k_{2}\right\}$, then $k_{1}=k_{2}$

(ii) If $e \leq \min \left\{k_{1}, k_{2}\right\}$, then $\operatorname{gcd}\left(\lambda\left(p^{e}\right), k_{1}\right)=$ $\operatorname{gcd}\left(\lambda\left(p^{e}\right), k_{2}\right)$, and we have a factorization of $\lambda\left(p^{e}\right)=u v$, where $u$ is the maximal divisor of $\lambda\left(p^{e}\right)$ relatively prime to $k_{1}$ and is also the maximal divisor of $\lambda\left(p^{e}\right)$ relatively prime to $k_{2}$. Moreover, for any divisor $d$ of $u$, ord $\mathrm{d}_{d} k_{1}=\operatorname{ord}_{d} k_{2}$.

Proof. By Corollary 20, we know that $G\left(p^{e}, k_{1}\right) \simeq G\left(p^{e}, k_{2}\right)$ if and only if $G_{i}\left(p^{e}, k_{1}\right) \simeq G_{i}\left(p^{e}, k_{2}\right), i=1,2$. But $G_{1}\left(p^{e}, k_{i}\right) \simeq$ $G\left(C_{(p-1) p^{e-1}}, k_{i}\right)$ if $p$ is odd, and $G_{1}\left(2^{e}, k_{i}\right) \simeq G\left(C_{2} \oplus C_{2^{e-2}}, k_{i}\right)$, where we use $C_{m}$ to denote the cyclic group of order $m$.

So we have $V_{p}\left(G_{2}\left(p^{e}, k_{1}\right)\right)=V_{p}\left(G_{2}\left(p^{e}, k_{2}\right)\right)$ and $p^{r} \| \operatorname{gcd}\left(\lambda\left(p^{e}\right), k_{i}\right)$ for some $r$. The proof is complete by Lemma 18 and Theorem 3.

\section{Data Availability}

No data were used to support this study.

\section{Conflicts of Interest}

The authors declare that there are no conflicts of interest regarding the publication of this paper. 


\section{Acknowledgments}

This research was supported by the Guangxi Natural Science Foundation (2015 GXNSFBA139012) and Inner Mongolia Natural Science Foundation (2018MS01017).

\section{References}

[1] A. V. Kelarev, Graph Algebras and Automata, Marcel Dekker, New York, NY, USA, 2003.

[2] A. Kelarev, J. Ryan, and J. Yearwood, "Cayley graphs as classifiers for data mining: the influence of asymmetries," Discrete Mathematics, vol. 309, no. 17, pp. 5360-5369, 2009.

[3] W.-S. Chou and I. E. Shparlinski, "On the cycle structure of repeated exponentiation modulo a prime," Journal of Number Theory, vol. 107, no. 2, pp. 345-356, 2004.

[4] C. Lucheta, E. Miller, and C. Reiter, "Digraphs from powers modulo p," The Fibonacci Quarterly, vol. 34, no. 3, pp. 226-239, 1996.

[5] T. D. Rogers, "The graph of the square mapping on the prime fields," Discrete Mathematics, vol. 148, no. 1-3, pp. 317-324, 1996.

[6] L. Somer and M. Kržek, "On a connection of number theory with graph theory," Czechoslovak Mathematical Journal, vol. 54, no. 2, pp. 465-485, 2004.

[7] L. Somer and M. Kržek, "Structure of digraphs associated with quadratic congruences with composite moduli," Discrete Mathematics, vol. 306, no. 18, pp. 2174-2185, 2006.

[8] L. Szalay, "A discrete iteration in number theory," BDTF Tud Közl, vol. 8, pp. 71-91, 1992 (Hungarian).

[9] B. Wilson, "Power digraphs modulo n," The Fibonacci Quarterly, vol. 36, no. 3, pp. 229-239, 1998.

[10] L. Somer and M. Kržek, "On semiregular digraphs of the congruence $x^{k} \equiv y(\bmod n)$," Commentationes Mathematicae, vol. 48, no. 1, pp. 41-58, 2007.

[11] W. Carlip and M. Mincheva, "Symmetry of iteration graphs," Czechoslovak Mathematical Journal, vol. 58, no. 1, pp. 131-145, 2008.

[12] G. Deng and P. Yuan, "On the symmetric digraphs from powers modulo n," Discrete Mathematics, vol. 312, no. 4, pp. 720-728, 2012.

[13] Y. Meemark and N. Wiroonsri, "The quadratic digraph on polynomial rings over finite fields," Finite Fields and Their Applications, vol. 16, no. 5, pp. 334-346, 2010.

[14] Y. Meemark and N. Wiroonsri, "The digraph of the $k$ th power mapping of the quotient ring of polynomials over finite fields," Finite Fields and Their Applications, vol. 18, no. 1, pp. 179-191, 2012.

[15] G. Deng and P. Yuan, "Isomorphic digraphs from powers modulo p," Czechoslovak Mathematical Journal, vol. 61, no. 3, pp. 771779, 2011.

[16] G. Deng and P. Yuan, "On the structure of $G(H, k)$," Algebra Colloquium, vol. 21, no. 2, pp. 317-330, 2014.

[17] M. Kržek, F. Luca, and L. Somer, 17 Lectures on the Fermat Numbers, From Number Theory to Geometry, vol. 9, SpringerVerlag, New York, NY, USA, 2001.

[18] L. Somer and M. Kržek, "On symmetric digrphs of the congruence $x^{k} \equiv y(\bmod n)$," Discrete Mathematics, vol. 309, no. 8, pp. 1999-2009, 2009.

[19] J. Kramer-Miller, "Structural properties of power digraphs modulo n," in Proceedings of the 2009 Midstates Conference for
Undergraduate Research in Computer Science and Mathematics, pp. 40-49, Oberlin College, Department of Computer Science, Ohio, USA, 2009. 


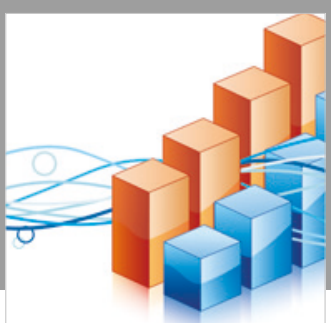

Advances in

Operations Research

\section{-n-m}
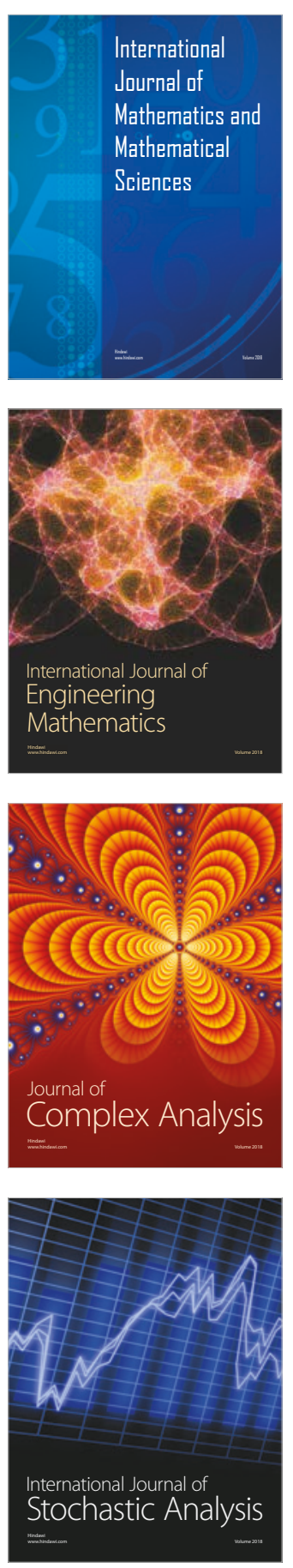
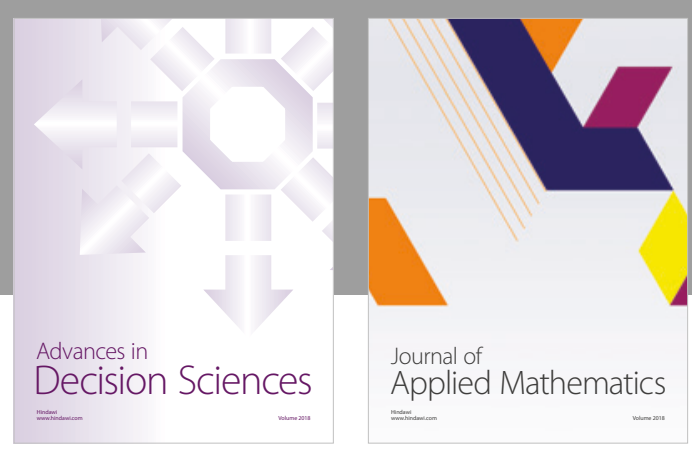

Journal of

Applied Mathematics
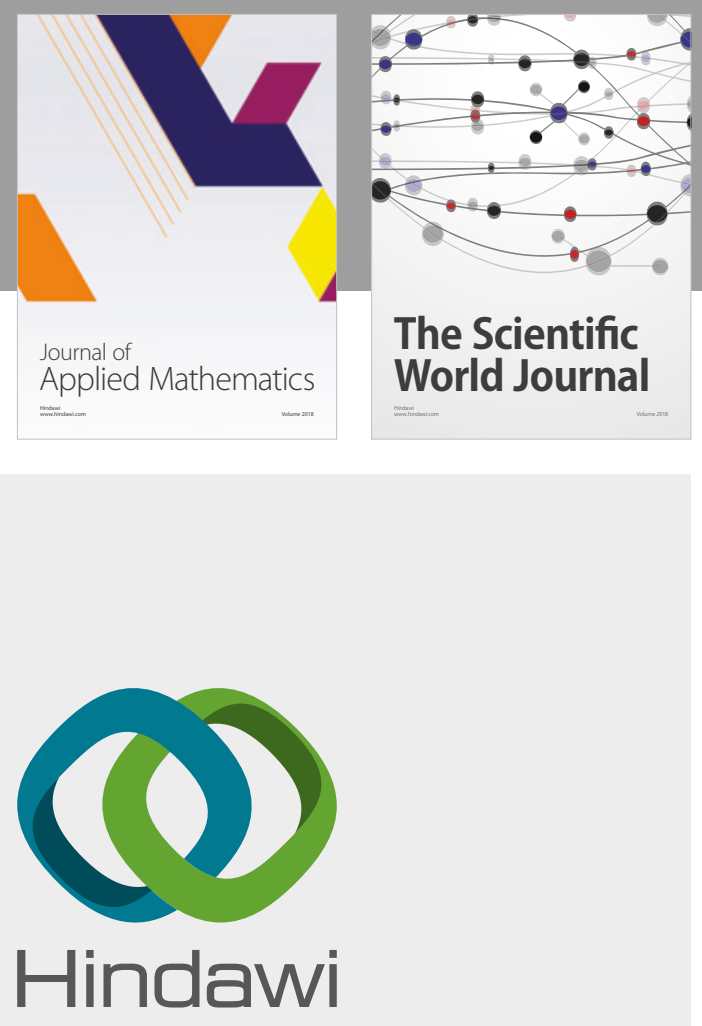

Submit your manuscripts at

www.hindawi.com

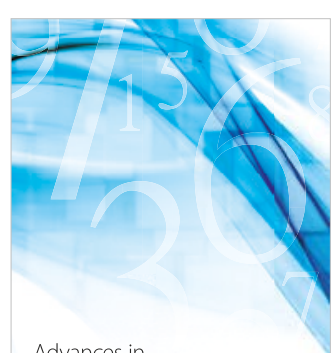

Advances in
Numerical Analysis
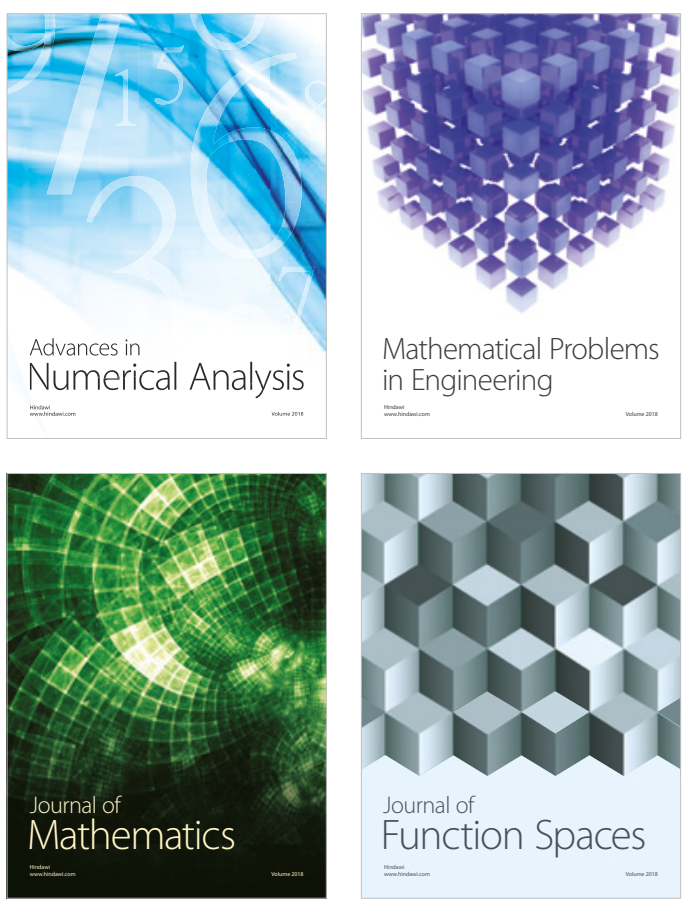

Mathematical Problems in Engineering

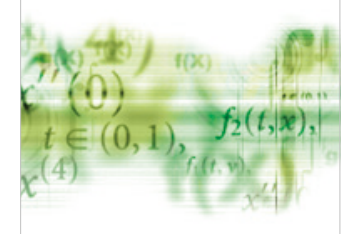

International Journal of

Differential Equations

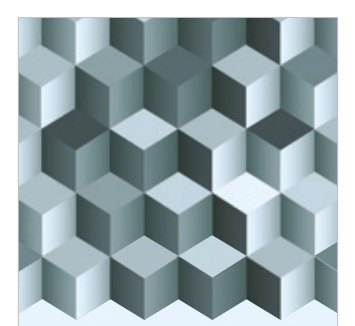

Journal of

Function Spaces

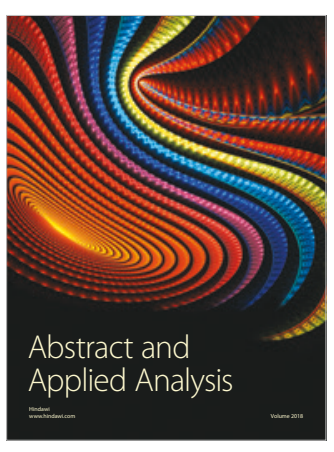

The Scientific

World Journal

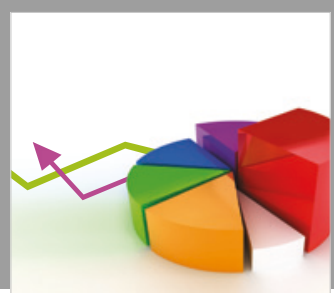

Journal of

Probability and Statistics
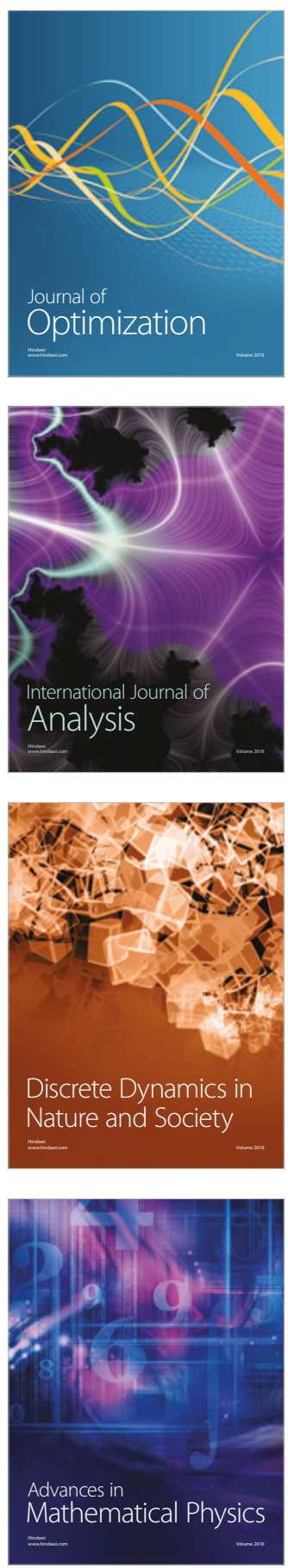